\section{Toronto-Sunnybrook Regional Cancer Centre Home Oncology Model Evaluation (H.O.M.E.) Pilot Program}

\author{
By Dianne E. Harrison and Margaret I. Fitch
}

\section{Abstract}

This paper describes the program development and evaluation experience at the Toronto-Sunnybrook Regional Cancer Centre between September 1990 and September 1992, with the Home Oncology Model Evaluation Pilot Program (H.O.M.E.). This paper demonstrates that the H.O.M.E. was clinically feasible, that no situation arose to jeopardize patient safety and that patient treatment was not compromised by treating chemotherapy patients in the home environment. The program proved to be a benefit for individuals who were physically debilitated but who wished to continue their chemotherapy at home. The authors believe that the program design utilized is a model which can be used as a basis for planning home chemotherapy in other communities.

Note: The program was made possible through the generous contribution of a private benefactor.

The option of receiving short-term infusion or intravenous push chemotherapy at home was not available in Canada until recently. In October 1991, the Toronto-Bayview Regional Cancer Centre, subsequently renamed the Toronto-Sunnybrook Regional Cancer Centre (T-SRCC), in conjunction with Home Care of Metropolitan Toronto and the St. Elizabeth Visiting Nurses' Association launched the Home Oncology Model Evaluation Pilot Program. The goal of the program was to develop a model for the delivery of chemotherapy in the home that might have applicability in other communities. This paper will provide a description of the program design and its evaluation at the end of the first nine months.

\section{Background and trends in cancer care}

By the year 2001, the trends of an aging and growing population will result in 60,000 newly diagnosed cancer patients annually in Ontario. This is a projected $50 \%$ increase over a 10-year interval

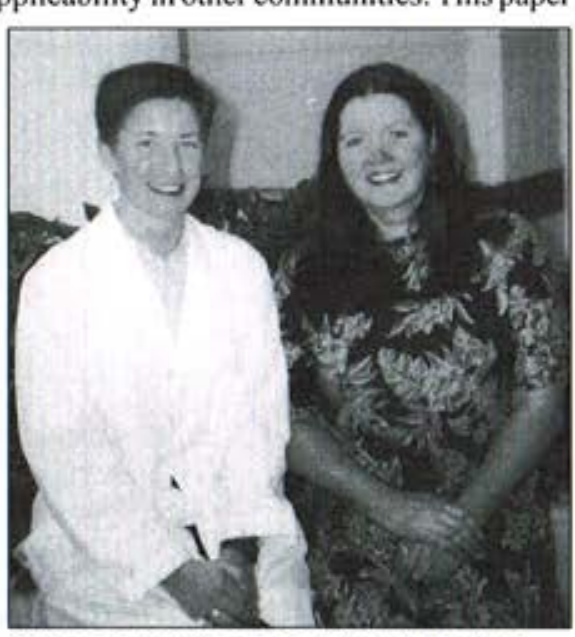

Dianne Harrison and Margaret Fitch
(NCIC, 1990). The increasing number of individuals diagnosed with cancer will create a proportionate demand upon health care resources including treatment services as well as support, rehabilitation and palliative care services (Cancer 2000, 1992).

The impact of a cancer diagnosis and its treatment produces a burden of suffering for individuals and their families which spans the physical, emotional and social realms. A cancer diagnosis is still perceived by many as a "death sentence". Both the disease and its treatment engender feelings of anxiety, loss of control and helplessness. An individual may experience physical changes (eg, anorexia, loss of hair, loss of weight, loss of body limb or function) and side effects (eg, nausea, vomiting, fatigue, pain). Such alterations carry the potential to change family roles and relationships, work responsibilities and communication with others. Clearly, cancer and its treatment can have a significant impact on quality of life.

Given the current level of concern regarding escalating health care costs, the potential for not meeting the requirements for cancer care are of grave concern. Currently, one strategy initiated to reduce health care costs is that of providing cancer chemotherapy on an outpatient basis in the community.

Furthermore, advances in science and technology have led to the design of new equipment which patients' family members can use safely (Benamed et al., 1985) and new pharmacological agents which make symptom management effective on an outpatient basis. The overall costs associated with care delivery can be reduced by moving care delivery to outpatient clinics and to patients' homes (Home IV, 1983; Nirenberg et al., 1981; Wodinsky et al., 1987).

\section{Growth of home chemotherapy}

The growth in home delivery of chemotherapy in the United States resulted from experiences with programs which provided intravenous infusions, parenteral nutrition or antibiotic therapy. The need for home chemotherapy was seen as a solution for patients with transportation

\section{CENTRE RÉGIONAL DU CANCER TORONTO-SUNNYBROOK PROGRAMME PILOTE D'ÉVALUATION DU MODĖLE DE SOINS ONCOLOGIQUES À DOMICILE (H.O.M.E.)}

\section{ABRÉGÉ}

Cet article décrit l'élaboration et l'évaluation du programme pilote H.O.M.E. au centre régional du cancer de Toronto-Sunnybrook qui s'y sont déroulées entre septembre 1990 et septembre 1992. L'article démontre que le programme H.O.M.E. est réalisable du point de vue clinique, qu'aucune situation n'est venue mettre en danger la vie des patients et que le traitement n'a nullement souffert du fait d'être administré à domicile. Le programme s'est avéré bénéfique pour tous ceux et celles qui étaient diminués physiquement mais voulaient poursuivre la chimiothérapie à domicile. Les auteures estiment que la conception de programme utilisée est un modèle qui peut servir à planifier la chimiothérapie à domicile dans d'autres collectivités.

Remarque: le Programme a pu être offert grâce à la généreuse contribution d'un bienfaiteur du secteur privé

Dianne E. Harrison, RN, MScN, is currently Clinical Nurse Specialist Oncology - Breast at Women's College Hospital in Toronto. Margaret I. Fitch, RN, PhD, is currently Head, Division of Oncology Nursing and Oncology Nurse Researcher at Toronto-Sunnybrook Regional Cancer Centre, North York, Ontario. 
problems, poor ambulation and lengthy treatments (Joseph et al, 1986) as well as patients who required long-term therapy with frequent hospitalization and separation from their normal psychosocial environment (Schlag et al, 1988). The intention in offering chemotherapy programs in the home is to maintain or enhance quality of life without diminishing the standards of tumour therapy.

The consumers of health care, to a great extent, encouraged and supported the movement toward community-based care (Esparza et al, 1989; Garvey, 1987). When given choices regarding the setting for care delivery, consumers select outpatient facilities because of quality of life, convenience and economics. For example, Vinciguerra et al (1980; 1986) described comprehensive home cancer care which reduced the amount of time advanced cancer patients spent in the hospital from $70 \%$ to $15 \%$. A third of these patients receiving home cancer care did not require hospitalization at all. Furthermore, the patients at home had access to family and friends, the continuation of normal activities and the comfort and familiarity of their own homes (Vinciguerra et al, 1980). Malone et al. (1986) reported that 15 gynaecological patients who received chemotherapy at home preferred that venue because of its increased privacy and convenience over the hospital setting. In addition, both Malone et al (1986) and Magid et al (1989) reported patients felt less anxiety in their own home environments.

Home chemotherapy programs in the United States use various organizational models. Fundamental to the majority are: (a) the use of oncology nursing experts to provide the chemotherapy and (b) mechanisms for quick access to medical expertise. Programs vary in their selection criteria for entrance, however, most use patient performance status and availability of support in the home as key criteria.

\section{Evaluation of home chemotherapy}

Anecdotal evidence suggests there are benefits of home chemotherapy programs that include enhanced patient comfort, family and patient convenience, quality of life and time savings. There is also reduction of drug toxicity, patient anxiety and exposure to hospital acquired complications (infection, injury, errors). However, disadvantages have also been identified which include lack of access to emergency response teams, disruption of family routines, increased responsibility upon the family and reduced access to the support of fellow patients at an outpatient clinic or hospital facility. To date, few of these programs are reporting systematic evaluations of home chemotherapy. However, some empirical evidence is contained in reports which contrast inpatient (hospital) versus community (home) delivery programs and demonstrate that there are benefits from chemotherapy delivery in the home.

Vinciguerra et al. (1986) did report a comparison of home versus hospital care for advanced cancer patients receiving antineoplastic chemotherapy. Medical benefits observed for the home treatment group included decreased narcotic analgesic requirements, numbers of hospitalizations and length of hospital stay. Furthermore, Vinciguerra et al. (1986) indicated that patient and family acceptance of the home treatment was excellent.

Mahmud et al. (1986) also assessed the feasibility of providing active cancer treatment to chemotherapy patients at home. Although actual rates/numbers were not reported, there was clear evidence of reduced side effects, decreased anxiety and avoidance of prolonged hospitalizations.

Furthermore, no major complications were encountered and patients demonstrated increased performance status.

Home chemotherapy was compared with hospital chemotherapy in 15 women who received their initial course of chemotherapy in hospital with subsequent courses at home (Malone et al, 1986). There were no extravasations or anaphylactic reactions reported for the 74 courses given at home. In the subjective reaction to therapy which was assessed by questionnaire, patients indicated that they preferred treatment at home primarily because of the personal care $(36 \%)$ and the convenience $(50 \%)$.

Schlag et al. (1988) reported on 64 patients with metastatic cancer receiving continuous infusion chemotherapy in their homes. The psychological evaluation found patients believed they were able to maintain a satisfactory quality of life. The most significant advantages according to the patients were: Avoidance of hospitalization, the maintenance of continuity of family life and the possibility of maintaining occupational or recreational activities.

The acceptance and adjustment to inpatient or outpatient continuous intravenous infusion chemotherapy was studied in 22 head and neck patients who received three to four cycles of induction chemotherapy and a five-day continuous intravenous infusion (CVI) (Magid et al., 1989). The 11 patients who received chemotherapy at home reported less anxiety at home as compared to the hospitalized sample. The individuals who opted for home delivery were, overall, more educated and had less physical impairment. Those who declined the home delivery cited the following reasons for their decision: Both inability to manage problems and fear of malfunctioning equipment ( $7 / 8$ patients), inability to participate in home activities (6/8 patients), physical difficulty wearing the infusion pump ( $5 / 8$ patients) and not having easy access to medical staff ( $5 / 8$ patients).

\section{Program planning}

Based on the literature reviewed at the onset of program planning, home chemotherapy was identified as a desirable development in the delivery of cancer care in the Metropolitan Toronto Region by the planning committee. There seemed to be more advantages for the patient to receive chemotherapy at home than in the hospital. However, it was not clear whether there were more advantages to receiving chemotherapy at home rather than attending an outpatient clinic.

The planning phase for the H.O.M.E. pilot program occurred between September 1990 and October 1991 under the auspices of a joint steering committee (see Table One for steering committee membership). Steering committee meetings were held monthly and chaired by the director of nursing for T-SRCC or the oncology clinical nurse specialist (CNS). Table Two outlines the critical steps in the design of the clinical model. The steering committee continued to meet following implementation in order to guide decisions about changes to the program and to provide a resource for ensuring unbiased decisions regarding specific patient referrals. The home chemotherapy program was designed with the following outcomes as its mission:

(1) The patient's quality of life is enhanced/maintained;

(2) The impact of symptoms/side effects is decreased;

(3) The patients and family members are satisfied with the services provided;

(4) The patient's treatment is not compromised;

(5) The health care professionals associated with the program are satisfied with the program;

\section{Table One: Members of the steering committee}

- Director of Nursing for T-SRCC;

- Vice-President for the Metropolitan Toronto Home Care Program;

- Branch Supervisor from Home Care of Metropolitan Toronto;

- Medical Oncologist from T-SRCC;

- Oncology Nurse Researcher for T-SRCC and Sunnybrook Health Science Centre (SHSC);

- Administrative Nurse Clinician for the T-SRCC chemotherapy unit;

- Administrative Nurse Clinician for day procedures and the narcotic infusion pump program at T-SRCC;

- Pharmacist for T-SRCC and SHSC;

- Two lay community representatives;

- Clinical Consultant from the St. Elizabeth Visiting Nurses;

- Palliative Care Coordinator from Providence Centre;

- SHSC Home Care Liaison Nurse.

Members added to the steering committee in

September of 1991 were:

- Oncology Clinical Nurse Specialist (CNS) as the Program Coordinator;

- Two oncology nurses from the St. Elizabeth Visiting Nurses. 
(6) The linkages between T-SRCC and the community (eg, Home Care, St. Elizabeth's Visiting Nurses Association, Providence Centre) are enhanced.

The fundamental responsibility for treatment decisions rested with T-SRCC staff (physicians, chemotherapy nurse, oncology CNS) while specially-trained visiting nurses (St. Elizabeth Visiting Nurses Association) administered the pre- and post-antiemetics and the chemotherapy medications to the patient at home. Home Care of Metropolitan Toronto served in its role of coordination of all home services.

Education and subsequent certification of the St. Elizabeth Visiting Nurses took place in the outpatient oncology unit, under the direction of the chemotherapy administrative nurse clinician.

\section{Program structure and process}

During the pilot phase, an oncology CNS was coordinator for the program. Patient referrals to the home chemotherapy pilot program were generated by the physicians, chemotherapy and primary nurses, or the oncology CNS for the program. Referrals generated by non-physicians were triaged by the oncology CNS and then discussed with the relevant physician. The flow for referral to the program is illustrated in Figure One.

Eligibility criteria for acceptance into the home chemotherapy pilot program included an "expressed/demonstrated difficulty getting to and from clinic because of debilitated status due to progressive disease or concurrent health problems or administration of highly toxic therapy which would require a period of close follow-up for symptom management at home." Exclusion criteria included residents who: (1) lived outside the Metropolitan Toronto catchment area; (2) required frequent physician contact in clinic or hospital; (3) were prescribed no chemotherapy requiring admixture immediately prior to treatment (i.e., Nitrogen Mustard); were enrolled in Experimental or Phase 2 chemotherapeutic agents; or ${ }^{*}$ Etoposide, ${ }^{*}$ Cisplatin, ${ }^{*}$ Bleomycin

Table Two: Critical steps in the design of the clinical model

- establish eligibility criteria;

- determine referral and care delivery mechanisms

(see Figure One - Patient Flow);

- identify clinical and decision-making responsibilities/job descriptions;

- develop documentation records (including consent form and ongoing nursing assessment checklist);

- detail medical directives;

- design program specific patient/family education materials;

- design community nurse orientation;

- research/evaluation questions and analysis;

- develop a data collection timeline;

- develop patient, family and chemotherapy treatment nurses' satisfaction evaluation questionnaire;

- establish contract with courier company for the situations where a family member or friend would be unable to pick up the chemotherapy at the clinic;

- establish contract with MDS labs to collect blood specimen two days prior to chemotherapy administration, with same-day reporting and weekend service;

- present program to all oncology-related services personnel;

- meet with medical oncologists to facilitate "buy-in" as well as to procure input;

- define parameter by which the CNS could approve the blood work prior to chemotherapy administration;

- establish weekly clinical rounds as start-up for program implementation;

- continue monthly steering committee meetings to identify trends and issues and assist with overall programmatic decisions;

- investigate computer software programs for data entry and analysis. (*prior to January 20, 1992) and L'asparaginase; (4) had a total treatment time greater than three hours; (5) had ANY documented anaphylactic reactions to antineoplastic agents. The process for screening patients for the pilot program was the responsibility of the oncology CNS.

The patients always received at least one course of the chemotherapy in the clinic before it was delivered in the home setting. Meanwhile, a team approach was taken to patient education. Patients and their families received the usual education from their primary nurses and chemotherapy nurse consultants regarding chemotherapy and its side effects while the initial teaching about the home chemotherapy program was conducted by the oncology CNS. Ongoing education was conducted by the visiting nurse in the home.

Ongoing communication between the oncology CNS and the patient care team members was maintained in several ways. The CNS met weekly with the St. Elizabeth Visiting Nurses, Home Care and Providence Centre representatives, clinic pharmacy supervisor and the chemotherapy administrative nurse clinician or her delegate. After the first four weekly meetings were held, the meeting frequency then changed to monthly. Close and regular communication (sometimes daily when a patient was receiving treatment in the home) was maintained between the St. Elizabeth Visiting Nurses, the oncology CNS and the Home Care head office representative for the program.

Once a patient had been referred and screened for eligibility for the program and informed, the oncology CNS obtained the patient's consent. Once consent was obtained, the referral was forwarded to Home Care, who then assigned the case and sent the information to the selected visiting nurse agency. The home visits were made by one of two specially-trained nurses from the St. Elizabeth Visiting Nurses Association.

The oncology CNS was responsible to: Complete the written Home Care referral and obtain the physician's approval and signature; ensure that the consent for the program was obtained; inform the clinic primary nurses and the chemotherapy nurses of their patients' experiences; attend return visits to clinic to process plans for the next home treatment and answer the patients' questions with the physician; inform the family physicians if their patients were admitted or discharged from the pilot program; inform the oncology physicians of patient outcomes following treatment administration or nursing intervention, prior to the patient's return to clinic (so their information would be complete); circulate current referral/refusal patient lists to the medical oncologist representative for the program and other key individuals; and make both verbal and written presentations about the program to the oncology community.

The visiting nurses who administered the chemotherapy routinely carried an adverse reaction kit provided by the clinic pharmacy and followed the medical directives of the clinic for adverse reactions. These medical directives were adapted for the home setting. Medical directives detailed actions to be taken for reactions such as: Acute anaphylaxis, extrapyramidal, extravasation, local hypersensitivity, hypertension, and hypotension. The clinic's guidelines for the disposal of antineoplastic waste were also followed. Hazardous chemotherapy waste containers were left in the patient's homes. When these containers were full, they were brought back by the patient to the next scheduled clinic visit and new containers were provided.

The patient's blood was taken in the home by an outside laboratory. The blood results were faxed from this laboratory to the oncology CNS. The oncology CNS had parameters from the physicians on which the chemotherapy could be approved without physician consultation. The oncology CNS notified the clinic pharmacy and the patient/family or friend, once the chemotherapy treatment was "approved". This "approval" meant the pharmacy could prepare the chemotherapy as previously written by the physician on the order sheet. Medications were picked up by a family member or friend, at a time pre-arranged with the clinic pharmacy. When a patient did not have a family member or friend, the medications were couriered to the patient's home. 
Figure One: Patient Flow for Participation in H.O.M.E. Program

( $*$ See explanatory notes for details)

T-BRCC nurse approaches patient in clinic to determine: Is the patient interested in hearing about the program?

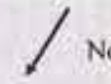

Refusal Noted

Reason Documented.

MD Decision Noted.
Reason Documented. No

Refusal Noted

Reason Documented: No

HC Decision Noted.
Reason Documented.

$\mathrm{HC}$ informs $\mathrm{CNS}$, who

informs patient and

provides a date and

time for return to

the clinic.
Borderline Results, or

Patient Unwell, Noted.

CNS consults with

Onc MD

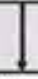

No chemo at home

CNS informs patient and

CTN, and provides a

date and time for return

to the clinic.

\section{EXPLANATORY NOTES}

Note "A": Patient eligibility criteria:

Expressed/demonstrated difficulty getting to and from clinic because of debilitated status duc to progressive disease or concurrent health problems or receiving highly toxic therapy requiring a period of close follow-up symptom management at home.

Note "B" - Legend:

- H.O.M.E. - Home Oncology Model Evaluation

- TBRCC - Toronto-Bayview Regional Cancer Centre

(now T:SRCC)

- CNC - TBRCC Chemotherapy Nurse Consultants

- CNS - Clinical Nurse Specialist

- H.O.M.E. Program Coordinator

- IIC - Home Care Liaison Nurse for program

- CTN-Community Treatment Nurses

- PCL - Palliative Care Liaison Nurse

- HCC - Home Care District Coordinator
Yes

CNS contacted by T-BRCC nurse. CNS reviews chart. ("Notes "A" \& "B")

CNS discusses patient with One. MD including: desired frequency of physician contact (t.e.: clinic visits);

pre-written chemo orders "pending results". Does MD find the patient suitable?

Yes

CNS meets/phones patient to provide program specific information and further assess patient suitability.

Chemo pick-up issue resolved. Is the patient still interested?

Yes

CNS calls HC for referral

HC calls the patient within 3 days. Is the patient eligible from HC's perspective?

Yes

HC informs CNS, who calls patient and asks them to bring a family member or friend, who will be available during home chemo times, to the next elemo in the clinic (i.e.: I'st "Program" chemo)

CNS notifies HC of I'st "Program" chemo date.

Patient "on program" receives next chemo at TBRCC

- Patient receives usual chemo teaching by TBRCC nurses.

- CNS repeats "Program specific" information to patient while at elinie for chemo, and provides written copy.

- Patient is admitted to HC program, given drug card and first set of supplies by HC,

CNS informs HC of date for first chemo at home, and HC arranges blood work to be drawn by MDS labs, 2 days prior to chemo with carly $A . M$. draw and same day results. HC informs patient of blood collection date and time for "first program chemo at home". Afterwards patient aware. (HCC arranges further blood collections)

Lab Day MDS faxes results to CNS

CTN calls patient to ensure blood drawn and assess patient status (eg: elevated temperature). CTN notifies CNS by 1500 hours. Results acceptable and patient feeling OK?

Yes

Chemo orders obtained from Onc MI

- Copy of orders to pharmacy to be attached to chemo bag(s) so CTN can verify.

- CNS documents on T-BRCC flow sheet re: home chemo.

- Patient aware to call T-BRCC Pharmacy for pick-up time

- CTN notifies patient of expected time of arrival for giving home chemo.

\begin{tabular}{|l|}
\hline Day ofHome Cheme \\
\begin{tabular}{|l|} 
Pt aware of time to expect CTN \\
Teaching reviewedreinforeed by CTN \\
Pre-chemo, chemo \& post-chemo given.
\end{tabular} \\
\hline - Assess need for additional nursing visits. \\
\hline - Ongoing coordination of home care by district coordinator at head office of MTHC. \\
\hline - Ongoing coordination of oncology care by T-BRCC and community team. \\
\hline
\end{tabular}

"Palliative component introduced on case by case basis by CTN to patient. 


\section{Evaluation of the pilot program}

Evaluation of a program should answer two basic questions: (1) Did the program operate as it was designed to operate, i.e. to deliver the desired service in the expected fashion? (2) Did the delivery of the service have the desired effect on the client? Evaluation of the delivery of chemotherapy in the home was seen as a critical component to our pilot program. This would enable future planning to be based on reliable, valid information concerning the effectiveness of the program.

It was anticipated that ultimately the program's outcomes would be achieved to a greater extent, for some individuals, by home chemotherapy delivery, than by the existing approach of visits to the outpatient facility or hospital, for chemotherapy. This question would be best answered by running a randomized clinical trial. The evaluation, initiated with the pilot stage of the program, was designed to answer the following questions: (1) Was the H.O.M.E. pilot program clinically feasible? (2) Was the H.O.M.E. pilot program economically feasible? (3) Who was apt to benefit from such a program:? (4) Were the data required to conduct appropriate evaluations available? (5) What was learned from the pilot experience that needed to be applied in future program planning?

\section{Patients referred to the program - pilot program experience during initial nine months}

A total of 91 patients were referred to the pilot program during the first nine months. This total included a number of individuals $(n=32)$ whom staff nurses thought would be appropriate for the program but who lived outside the program's Metropolitan Toronto catchment area. These individuals were not reviewed further for eligibility or approached about the program.

Of the 59 remaining patients who were reviewed for eligibility, 24 did not meet one or more of the program criteria (see Table Three for a listing of the reasons for ineligibility). Thirty-five individuals, then, were deemed eligible for the pilot program. Physicians subsequently declined participation in five situations and patients declined participation in 14 situations. The reasons for physician non-participation were: A desire to see the patient each time chemotherapy was to be given $(n=2)$; few treatments remaining $(n=2)$; concern for the patient's risk of repeat occurrence of pulmonary edema $(n=1)$. The reasons patients provided for non-participation included: Preference for the clinic $(n=13)$ and change of mind for treatment at home with the recognition that the disease and subsequent treatment were palliative $(n=1)$.

In total, 16 individuals met all criteria and agreed to participate in the pilot program. Eleven of those patients entered the program and received chemotherapy at home. The other five received their initial

Table Three: Twenty-four patients ineligible within Metropolitan Toronto boundaries

Reasons

Needed to be seen by oncologist prior to each chemotherapy

On excluded chemotherapy medications (prior to Jan. 20, 1992)

Referral for transportation problems

Hospitalized after referred \& subsequent discontinuation of chemotherapy

Condition very poor when referred \& treatment stopped same or next day

Treatment stopped

Chemotherapy changed to oral route

Reason for referral was an emotional debility

Reason for referral was patient obesity - MD subsequently changed mind "program" chemotherapy in the clinic but were unable to continue at home because their conditions deteriorated, they were subsequently hospitalized and/or they died.

\section{Patients who received chemotherapy at home}

The demographic characteristics of the 11 individuals who received chemotherapy at home are listed in Table Four. The five men and six women had an average age of 68 years, represented a variety of diagnostic sites (breast, lung, colon, rectum, bladder), and were on various chemotherapeutic protocols (see Table Five).

Length of time on the program ranged from 22 to 117 days with an average of 68 days. Both the length of time between protocols and the actual number of chemotherapies per cycle varied and therefore influenced the number of home visits per patient during his/her time on the program. Patients averaged six home chemotherapy visits while on the program. The range was a low of one visit over six weeks, to a high of 14 visits over 15 weeks. During their time on the program, patients averaged two clinic visits for assessment purposes. The range was a low of one visit over six weeks to a high of four clinic visits over 17 weeks.

The largest number of individuals who were on the pilot program and receiving chemotherapy at home at any one point was seven. The length of time required for home chemotherapy visits averaged 94 minutes with a high of three hours and a low of 40 minutes.

\section{Clinical course of patients on the program}

A range of clinical parameters (see Table Six) were monitored for each patient on the program by the St. Elizabeth Visiting Nurses who used a pre-chemotherapy nursing assessment checklist. A total of 63 administrations of chemotherapeutic agents occurred in the home. Of particular note was that there were no adverse reactions (eg, anaphylaxis, extravasation, hypertension, hypotension, respiratory difficulties). Patients experienced the side effects of nausea, minimal vomiting, mild mucositis, and diarrhea which were dealt with as they would have been had the patient experienced or reported these following chemotherapy at the clinic. Of note is that nausea and vomiting were uncommon occurrences for the home chemotherapy patients. One case was that of a female patient who had normally experienced nausea and vomiting for 48 hours prior to receiving chemotherapy at the clinic. This patient experienced no anticipatory nausea or vomiting when she received chemotherapy at home. In

Table Four: Demographics of patients who received chemotherapy at home
Male

5

Total patient population

Age

$$
\begin{array}{r}
\text { average } \\
\text { range }
\end{array}
$$
widowed

Marital status living at home with patient average range

$0-3$

Education level

Some grade school

Completed grade school

Some high school

Completed high school

Some college/university

Completed college/university

$0-3$

Completed graduate school married 4 
another case, a male patient who did not eat much for five to seven days around his three-day chemotherapy at the clinic expressed how much more relaxed he felt. He would be in the middle of enjoying a full breakfast when the visiting nurse arrived at his home to administer the chemotherapy,

\section{Patient/family responses to the program}

The program participants who had received a minimum of two cycles of chemotherapy at home, and one of their family members, were interviewed about their experiences with the program. Without exception, all described the program in positive terms, using words like "wonderful", "terrific", "excellent". Participants reported that having the chemotherapy at home "made it so easy for me", "fit right into my day" and "made it relaxing". In all instances, family members agreed.

All participants and family members indicated that the teaching they had received prepared them, yet they all stated they knew they could call for assistance if they experienced any problems. When asked if any changes ought to be made in the program, the suggestions that were offered were: To have the same nurse visit each time (SEVN patients have a "primary nurse" who is not the same nurse administering the chemotherapy) and to have the nurse check every day or so with the patient. Another concern mentioned by participants and family members related to the worry about receiving the right medication and dosage and about the instructions from the clinic having been communicated to the nurse giving the chemotherapy at home.

\section{Visiting nurses' responses to the pilot program}

The two nurses from the St. Elizabeth Visiting Nurses Association who administered the chemotherapy in the home were asked to respond in a written report about their experiences with the pilot program. Overall, the nurses said they were very satisfied with the pilot program. Both nurses agreed that the orientation program they had received at the clinic gave them an adequate knowledge base to prepare them for their roles. They also stated that they found the pilot program was very well organized and they experienced no disappointments.

Both nurses felt fortunate being part of this pilot program and "finally seeing patients receive chemotherapy in their home in a relaxing environment" and were "delighted to see how successful the program was for the patients in the control of vomiting pre- and post-chemotherapy". The nurses pointed out that the patient and family benefited by not having to go out to the clinic in a state of decreased energy/fatigue. The Home Care support in the program was perceived by the nurses to be excellent, as was the support and communication from all staff at the regional cancer centre.

Limitations of the pilot program were also identified by the visiting nurses. These limitations were: The present Metropolitan Toronto boundaries, the inclusion criteria related to physical debility, and that the chemotherapy supplies were not delivered to the patients' homes, but had to be picked up from the clinic by family or friends.

Table Five: Disease/treatment profile of patients who received chemotherapy at home

\section{MALE}

\begin{tabular}{|c|c|c|c|c|c|c|}
\hline Age & $\begin{array}{l}\text { Diagnosis } \\
\text { (Disease site } \\
\text {-primary) }\end{array}$ & $\begin{array}{l}\text { Site of } \\
\text { metastasis }\end{array}$ & $\begin{array}{l}\text { Functional } \\
\text { status - ECOG } \\
\text { at intake }\end{array}$ & $\begin{array}{l}\text { Functional status } \\
\text { - change while } \\
\text { on program }\end{array}$ & Treatment Protocol & $\begin{array}{l}\text { Access site } \\
\text { (IV, Port-a-cath) }\end{array}$ \\
\hline 61 & CA bladder & $\begin{array}{l}\text { Liver, It. pelvis, } \\
\text { rt. pubic area }\end{array}$ & 2 & 1 & $\begin{array}{l}\text { Cisplatin, Adriamycin, MTX, } \\
\text { Vinblastine, Ondansetron }\end{array}$ & IV \\
\hline 60 & $\mathrm{CA}$ lung & Adrenal & 2 & 1 & $\begin{array}{l}\text { VP-16, Cisplatin, Dexamethazone, } \\
\text { Ondansetron }\end{array}$ & IV \\
\hline 79 & CA colon & Liver & 2 & 3 & $5 \mathrm{FU}, \mathrm{FA}$ & IV Push \\
\hline 71 & CA lung & Lymph nodes & 2 & 1 & $\begin{array}{l}\text { Cyclophosphamide, Adriamycin } \\
\text { Vincristine, Cisplatin, VP-16, } \\
\text { Dexamethazone }\end{array}$ & IV \\
\hline 63 & CA colon & CA colon & 2 & 2 & $5 \mathrm{FU}, \mathrm{FA}$ & IV Push \\
\hline \multicolumn{7}{|c|}{ FEMALE } \\
\hline Age & $\begin{array}{l}\text { Diagnosis } \\
\text { (Disease site } \\
\text {-primary) }\end{array}$ & $\begin{array}{l}\text { Site of } \\
\text { metastasis }\end{array}$ & $\begin{array}{l}\text { Functional } \\
\text { status - ECOG } \\
\text { at intake }\end{array}$ & $\begin{array}{l}\text { Functional status } \\
\text { - change while } \\
\text { on program }\end{array}$ & Treatment Protocol & $\begin{array}{l}\text { Access site } \\
\text { (IV, Port-a-cath) }\end{array}$ \\
\hline 56 & CA breast & $\begin{array}{l}\text { Multiple bone } \\
\text { sites }\end{array}$ & 2 & 2 & Epirubicin, Dexamethasone & IV Push \\
\hline 102 & CA breast & None & 2 & 3 & Adriamycin, Prochlorperazine & IV Push \\
\hline 79 & CA colon & Liver & 2 & 1 & $\begin{array}{l}5 \text { FU, FA, Diphenhydramine } \\
\text { Metachlopramide }\end{array}$ & IV \\
\hline 73 & $\mathrm{CA}$ rectum & Lymph nodes & 2 & 2 & 5 FU, FA, Prochlorperazine & IV Push \\
\hline 65 & $\mathrm{CA}$ breast & Vertebral & 2 & 2 & $\begin{array}{l}5 \text { FU, MTX, Dexamethasone } \\
\text { Metachlopropamide }\end{array}$ & IV \\
\hline 36 & CA breast & $\begin{array}{l}\text { Pelvis, liver, } \\
\text { spine }\end{array}$ & 2 & 0 & $5 \mathrm{FU}, \mathrm{FA}$ & IV Push \\
\hline
\end{tabular}


Table Six: (Part of) Prechemotherapy and Ongoing Nursing Assessment Checklist for the H.O.M.E. Chemotherapy Program Note: Nursing interventions and evaluations and related patient strategies for ANY checklist item are documented on the SEVN chart.

FAX to at when completed.

PATIENT PROFILE

Name:

\begin{tabular}{|l|l|}
\hline DATE: & Telephone call: \\
\hline Reason for call: \\
\hline Home visit: \\
\hline Reason for visit: \\
\hline TOTAL PHONE TIME: \\
\hline TOTAL VISIT TIME: \\
\hline $\begin{array}{l}\text { Of phone/visit time, time spent on: } \\
\text { a) teaching }\end{array}$ \\
\hline b) support \\
\hline c) chemo (admin) \\
\hline d) other (specify) \\
\hline CTN Signature:
\end{tabular}

PART I: PHYSICAL STATUS

BP

Temp

Pulse

Resp

Weight (if possible)

HEMATOLOGY

WBC

$\mathrm{Hgb} / \mathrm{Hct}$

Platelets

Other

INTEG

Mucositis Rating*

Site

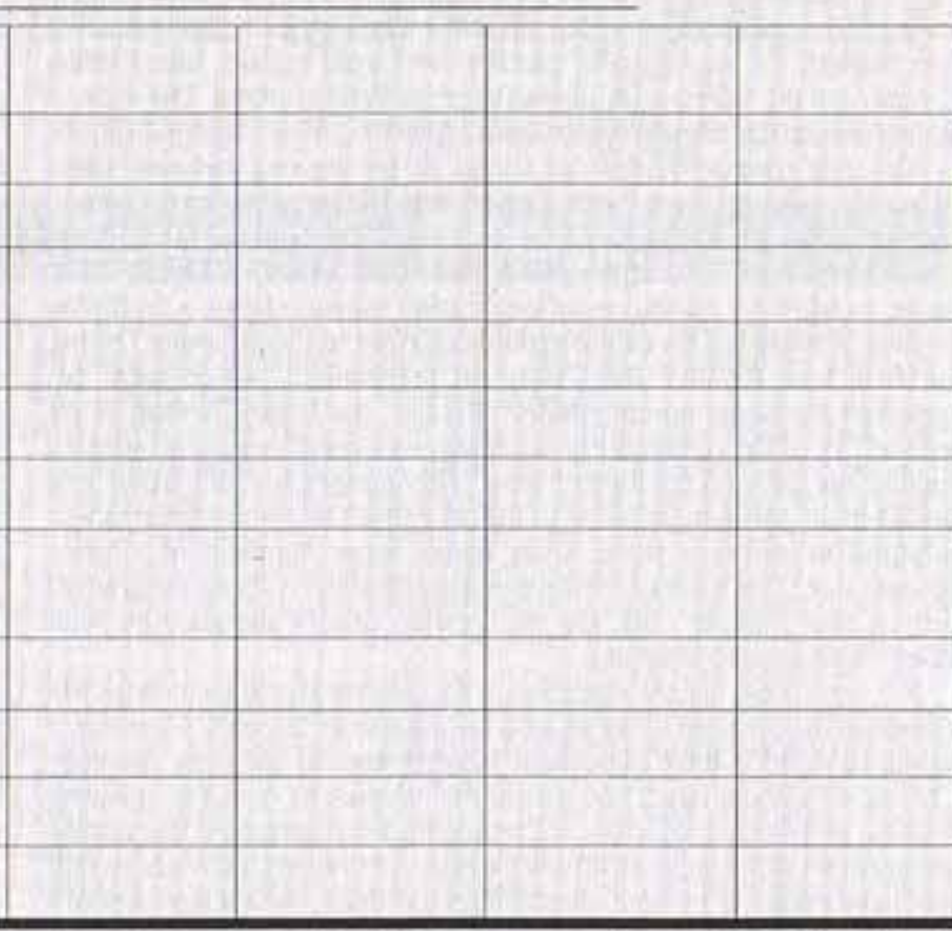

*Mucositis Rating Scale

0 - Pink, moist, intact mucosa, absence pain

+2 - Isolated ulcerations $+/$ - white patches

+1 - Generalized erythema $+/-$ pain

+4-Haemorrhage ulcerations

+3 - Confluent ulcerations, white patches

("Adapted from Engelking, C.H. and Steele, N.E. (1984). A model for pretreatment nursing assessment of patients receiving cancer chemotherapy. Cancer Nursing, 7(3), pp.203-212) 


\section{Discussion}

In summary, the Home Oncology Model Evaluation Pilot Program was found to be clinically feasible. There were no safety issues identified and treatment was not compromised in any cases. The program was a benefit for individuals who were physically debilitated, but who wished to continue their chemotherapy treatments at home. The economic feasibility could not be determined because relevant and specific cost data were not retrievable. The low numbers accrued to the pilot program resulted from: The limited number of medications approved for home administration during the first four months, the exclusion of patients who lived outside Metropolitan Toronto and the late stage of disease required before referral. The issue of the low accrual rate was a primary aspect of planning the next program phase.

The late disease stage was found to be limiting because some referred patients were well into the palliative treatment phase, consequently, their deterioration warranted hospitalization and the cessation of chemotherapy prior to their first scheduled treatment at home. In addition, the approved medication list was clearly a limitation because it excluded six of the patients referred to the program. During the first four months, they were on medications which were not approved for home administration. Finally, the largest limitation for accrual ( 32 of the referrals not accepted) was because patients resided outside the defined catchment area. This geographic limitation was altered in the subsequent phase of the program. The catchment area was enlarged to embrace those areas where large numbers of eligible patients live. This change required extension of the training program to include new nursing staff and the creation of new partnerships with other visiting nurse agencies.

It is anticipated that the extension of the catchment area boundaries will impact on the program by increasing the number of referrals. However, it should be noted that the pilot phase incorporated the use of an oncology CNS on a full-time basis. She sought out referrals by regularly attending "rounds" in the outpatient clinic and promoting the program. As the ongoing management of the program is now the responsibility of clinic nursing staff who have a number of other clinical responsibilities, the management of a high accrual may well continue to keep enrolment in the program low.

Surprisingly, a significant number of eligible individuals $(n=13)$ preferred not to have treatment in their home, but cited a preference for receiving the chemotherapy in the clinic. They indicated that treatment at the clinic was preferred because they enjoyed the visits and wanted to

\section{References}

Benamed, M., Carde, P., Laplanche, A., et al. (1985). Ambulatory Chemotherapy Under Continuous Perfusion by Portable Pump: applicable trial. Bull Cancer, 72:30-36.

Cancer 2000. (1992). The Proceedings of Cancer 2000. Toronto: Canadian Cancer Society.

Esparaza, D., Young, N., Luongo, J. (1989). Effective planning for office and outpatient chemotherapy administration. Seminars in Oncology Nursing, 5(2), 8-14.

Garvey, E. (1987). Current and future nursing issues in the home administration of chemotherapy. Seminars in Oncology Nursing, 3(2):142-147

Home IV Cancer Chemotherapy benefits to patients and hospital. Hospital Peer Review, 4:45-7, 1983.

Magid, D.M., Vokes, E.E., Schilsky, R.L., Guarnieri, C., Whaling, S., Weichselbaum, R., Panje, W. (1989). A Randomized Study of Inpatient versus Outpatient Continuous Intravenous Infusion Chemotherapy: Psychosocial Aspects. Selective Cancer Therapeutics, 5(3):137-145.

Mahmud, K., Vukelich, M.A., Londer, H., Manion, J., Levitan, A., Krengel-Kraus, J. (1986). A Mobile Cancer Clinic. Minnesota Medicine, 69(10):581-584

Malone, J., Kavanagh, J., Crosson, K., Streckfuss, B. (1986). Domiciliary Chemotherapy for Malignant Diseases. Eru J Gynae Oncology, VII(2):120-121. continue to come to the clinic as long as they were physically able. Furthermore, they did not want someone or the "clinic" in the home, and also preferred to have access to emergency back-up equipment which the clinic offers. It is possible that the clinic venue may well have provided a social network for these individuals as well as allowed them to separate the illness from the other parts of their life at home. One might also question the existence of the need to exert control over one's situation or to maintain a sense of independence by getting dressed and coming to the clinic. Although the reasons underlying the observed preferences need further exploration, it is clear that some people do not want to be part of a home chemotherapy program. Our approach to individuals must bear this in mind and leave the options with the consumer.

Other consumers of the pilot program were physicians. In order to "sell" the program to physicians, the oncology CNS and the administrative nurse clinician for the chemotherapy unit attended meetings of the medical oncologists to present information on the program, obtain oncologists' input and answer any questions. The medical directives and program materials were reviewed by the physicians prior to being utilized. This helped to garner their support. Feedback to specific physicians on how their patients were doing on the pilot program was part of the ongoing work of the oncology CNS, as was the provision of general information on the program's progress, to all physician staff. Regular program updates were printed in the clinic newsletter and presentations were made to various clinic administrative groups. This overall approach served to meet the challenge of marketing the program at the clinic.

\section{Conclusion}

The work of the steering committee for the H.O.M.E. pilot program represented a success story in collaborative planning. The expertise of all members was utilized, valued and respected. The end result was that of a well organized program which ran smoothly. The pilot program was found to be a model which could be continued at the clinic and used as a basis for planning home chemotherapy in other communities.

Based on the pilot phase evaluation and experience, the home chemotherapy program moved into the subsequent phase. The eligibility criteria and clinical protocols remained the same but the geographical boundaries were extended. In addition, Princess Margaret Hospital implemented the program as well. It is anticipated these changes will facilitate an evaluation with a larger number of patients and cost-benefit analysis.
NCIC, 1990. Discussion Paper on Cancer Control. National Cancer Institute of Canada Advisory Committee on Cancer Control. (Toronto, June, 1990).

Nirenberg, A., Cacavio, G.B., Caparros, B., et al. (1981). Ambulatory high dose cisplatinum (HD-DDP) with mannitol diuresis: a treatment system without renal, auditory or biochemical toxicity. Proc Am Soc Clin Oncol, 22:528 (Abstract C-766).

Schlag, P., Schwarz, A., Frohmüler (1988). Drug Delivery to Cancer Patients in Home Care: Experience with Intra arterial and Intravenous Chemotherapy. In Drug Delivery in Cancer Treatment II. Ed: L. Domellof. New York: Springer-Verlag.

Vinciguerra, V., Degnan, T., Deiner, J., Budman, D., Schulman, P., McCartney, J., O'Connell, M., Vargas, M. (1980). Home oncology medical extension: A new home treatment program. CA-A Journal for Clinicians. 30(3):182-185.

Vinciguerra, V., Deghan, T.J., Sciortino, A., O’Connell, M., Moore, T., Brody, R., Budman, D., Eng, M., Carlton, D. (1986). A Comparative Assessment of Home Versus Hospital Comprehensive Treatment for Advanced Cancer Patients. Journal of Clinical Oncology, 4(10):1521-1528.

Wodinsky, H., DeAngelis, C., Rusthoven, J.J., Kerr, I.G., Sutherland, D., Iscoe, N., Buckman, R., Kornijenko, M. (1987). Re-evaluating the cost of outpatient cancer chemotherapy. CMAJ, 137, 903-906. 